\title{
Notfallbehandlung eines epileptischen Anfalls mit Yamamoto Neuer Schädelakupunktur (YNSA) und Körperakupunktur - Falldarstellung zur Akupunktur in der Notfallmedizin
}

\author{
Thomas Schockert $^{\mathrm{a}, \mathrm{b}} \quad$ Frank Dittmar $^{\mathrm{c}} \quad$ Jochen M. Gleditsch $^{\mathrm{d}}$ \\ ${ }^{a}$ Fachbereich Chinesische Medizin, Universität Witten/Herdecke, \\ ${ }^{\mathrm{b}}$ Nideggen, \\ ${ }^{c}$ Diakonie Krankenhaus Marburg, Deutschland \\ ${ }^{\mathrm{d}}$ Wien, Österreich
}

\section{Schlüsselwörter}

Epilepsie · Bewusstlosigkeit · Koma - Atemstillstand · YNSA $\cdot$ Akupunktur $\cdot$ Notfallmedizin

\section{Zusammenfassung}

Hintergrund: Weltweit wird Akupunktur bereits erfolgreich in Krisengebieten und auch zunehmend häufiger supportiv in der Notfallmedizin angewendet. Methoden: Hier wird die Behandlung eines Patienten mit zerebralem Krampfanfall, Masseterkrampf, Bewusstlosigkeit und Atemstillstand durch YNSA und Körperakupunktur an den 3 Akupunkturpunkten YNSA-Yin-Basalganglien, Lenkergefäß 26 (Renzhong) und Dünndarm 2 (Qiangu = Punkt zur Kieferöffnung) beschrieben. Ergebnisse: Nach Durchführung der Akupunktur sistierte der Krampfanfall; der weiterhin bewusstlose Patient öffnete den Mund und hatte eine sichere Spontanatmung. Schlussfolgerung: Akupunktur hat in diesem Fall als supportives Therapieverfahren den Ablauf des Notfalleinsatzes erheblich erleichtert und dem Patienten rasche und sichere Hilfe verschafft.

\section{Einleitung}

Die Kategorisierung internationaler Rettungsdienstsysteme (RDS) wird traditionell anhand des jeweils eingesetzten Rettungsdienstpersonals vorgenommen [1]. $\mathrm{Zu}$ benennen sind folgende Systeme: Das Paramedic-System (Anglo-American

\section{Keywords}

Epilepsy · Unconsciousness · Coma - Respiratory arrest . YNSA - Acupuncture · Emergency medicine

\section{Summary}

Emergency Treatment of Epilepsy with Yamamoto New Scalp Acupuncture (YNSA) and Body AcupunctureAcupuncture in Emergency Medicine: A Case Report Background: Worldwide, acupuncture is used in conflict areas and increasingly also as a supportive measure in emergency medicine. Methods: In this case, the treatment of epilepsy, masseter cramp, unconsciousness and respiratory arrest by means of YNSA and body acupuncture with only 3 acupuncture needles is described. The 3 points used were YNSA basal ganglia point, renzhong and qiangu. Results: After application of the needles, the epileptic fit stopped, the unconscious patient opened his mouth and started breathing spontaneously. Conclusion: In this case, acupuncture simplified the emergency procedure as a supportive treatment method and provided the patient with fast and safe relief.

Emergency Medical System = AAS), das Mischsystem (Griechenland), das arztzentrierte System (Franco-German Emergency Medical System = FGS) und das Nurse-System (Niederlande). Deutschland, Belgien, Frankreich, Spanien, Portugal, Italien und Finnland gehören allesamt zu den arztzentrierten Systemen [2]. Das Unterscheidungskriterium ist

\section{KARGER \\ Fax +497614520714 \\ Information@Karger.de}

www.karger.com 
hier das Vorhandensein oder Nichtvorhandensein eines notfallmedizinisch und präklinisch tätigen Arztes oder Notarztes bzw. in Anlehnung an den Heidelberger Chirurgen Kirschner die Tatsache, ob «der Arzt zum Patient gebracht wird» (FGS) oder «der Patient zum Arzt gebracht wird» (AAS).

Die Möglichkeit, präklinische Notfallakupunktur zu praktizieren, bietet sich vor allem für arztzentrierte RDS wie z.B. die in Deutschland an, wo nicht wenige Notärzte über eine Akupunkturausbildung verfügen.

Ein wichtiges Argument für den Einsatz von Akupunktur in der Notfallmedizin kann ihre meist schnelle und zuverlässige Wirksamkeit sein. Insbesondere bei Einsatz der YNSA werden oft ein rascher Wirkungseintritt oder Sekundenphänomene beschrieben [3]. Dies bestätigt die Erfahrung der Autoren dieser Fallbeschreibung.

\section{Fallbericht}

Um 22:23 Uhr erhalten Rettungswagen (RTW) und Notarzt (NEF) die Einsatzmeldung: Bewusstlose Person, vermutlich zerebraler Krampfanfall. RTW und NEF treffen zeitgleich um 22:34 Uhr am Haus des Patienten ein. Das Haus des Patienten liegt in einem unzugänglichen Steilhang, der Fußweg zum Haus über eine schmale rutschige Treppe dauert ca. $3 \mathrm{~min}$.

\section{Methoden}

Im hier beschriebenen Fall sind folgende Akupunkturpunkte zum Einsatz gekommen: Der YNSA-Yin-Basalganglien-Punkt [4] (Abb.1) wurde zusammen mit dem Punkt Lenkergefäß 26 (Renzhong) [5] akupunktiert. Zusätzlich erhielt der Patient eine Akupunktur des Punktes Dünndarm 2 (Qiangu $=$ Punkt zur Kieferöffnung) [6].

\section{Anamnese}

Der Patient wurde von seiner Mutter krampfend, bewusstlos und nicht ansprechbar im Bett aufgefunden. Anamnestisch bekannt sei ein seit 1996 bestehender insulinpflichtiger Diabetes mellitus, das Auge links sei fast blind, der Patient habe mehrere Netzhautoperationen und Linsenoperationen über sich ergehen lassen müssen. Aktuell bestehe eine akute Sinusitis.

\section{Befunde}

In der Untersuchung des 22-jährigen Patienten zeigte sich eine Blickde viation beider Pupillen nach rechts oben. Aufgrund des Atemwegsinfekts waren die Atemwege des Patienten sehr verschleimt; das Atmen durch die Nase war aufgrund des zähen Sekrets nicht möglich.

Neurologische Untersuchung 22:40 Uhr: kein Öffnen der Augen, keine verbale Reaktion, keinerlei motorische Reaktion. Somit ergibt sich gemäß der Glasgow-Coma-Scale (GCS) ein minimaler Wert von 3, welcher als Ausdruck eines tiefen Komas zu werten ist.

Die Pupillen sind beidseits mittelweit und reagieren nicht auf Licht. Auffällig ist eine Blickdeviation beider Pupillen nach rechts. Der systolische Blutdruck beträgt $80 \mathrm{~mm} \mathrm{Hg}$ der Puls liegt bei 98/min und ist regelmäßig. Der Blutzucker ist mit $93 \mathrm{mg} / \mathrm{dl}$ unauffällig, die Sauerstoffsättigung wird nach $\mathrm{O}_{2}$-Gabe mit $99 \%$ gemessen. Im EKG zeigt sich ein $\mathrm{Si}$ nusrhythmus; die Lunge ist über allen Abschnitten belüftet. Auskultatorisch ist großblasiges Schleimrasseln über der Trachea und den Bronchien zu hören.

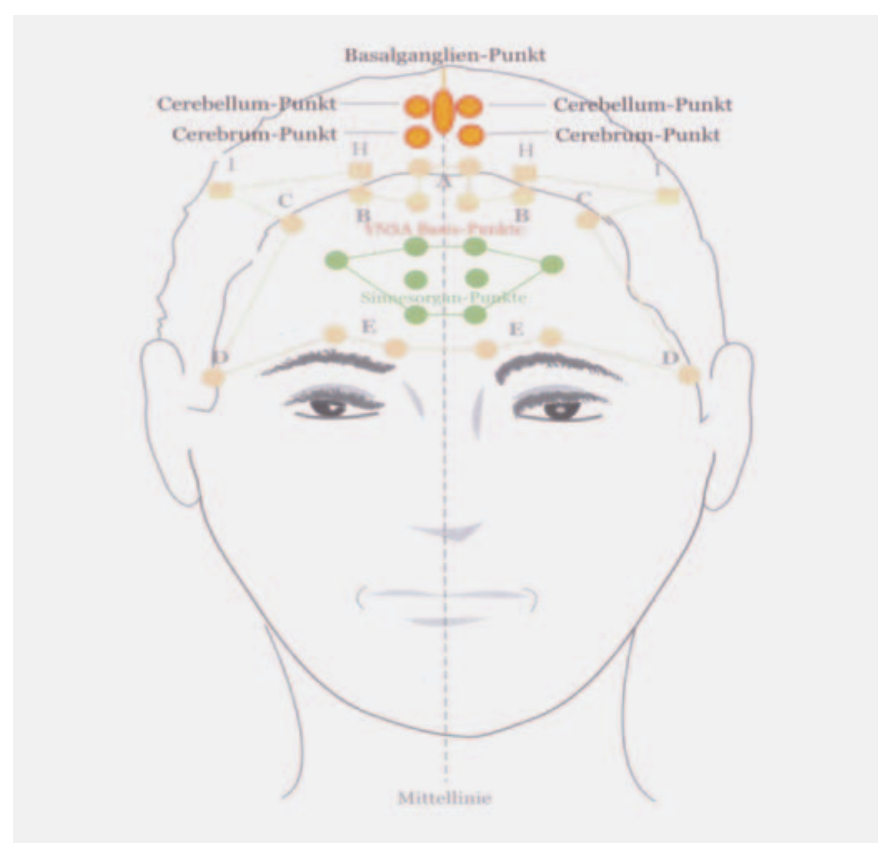

Abb. 1. YNSA-Yin-Basispunkte mit Basalganglienpunkt.

\section{Diagnosen}

Zerebraler Krampfanfall, Bewusstlosigkeit unklarer Genese, insulinpflichtiger Diabetes mellitus, Retinopathie, akute Sinubronchitis.

\section{Verlauf}

Der Patient wird, da er eine gute Spontanatmung durch den geöffneten Mund hat, zunächst mit einem intravenösen Zugang am linken Unterarm versorgt und mit $500 \mathrm{ml}$ Ringer-Lösung aufinfundiert. Er bekommt sofort Sauerstoff und beginnt erneut zu krampfen. Daraufhin erfolgt die intravenöse Gabe von fraktioniert $2 \times 0,5$ Ampullen Rivotril (Clonazepam). Der Patient reagiert zögerlich. Nach Sistieren der Myoklonien und Lagerung auf dem Tragetuch beginnt der Transport durch das enge Treppenhaus. Auf der engen Treppe hinab vom Dachgeschoss, in dem sich das Zimmer des Patienten befindet, setzt ein erneutes starkes Krampfen ein. Der Patient verkrampft insbesondere die Massetermuskulatur, krampft dann stärker; der Mund ist nicht zu öffnen und es kommt zum Atemstillstand. Die Rettungsassistenten erhalten die Anweisung zum Vorbereiten einer Intubation. Da der Notfallkoffer für den Transport zum RTW bereits wieder verschlossen worden ist und sich unterhalb der Treppe befindet, erfolgt der sofortige Entschluss zum Einsatz von Akupunktur.

Noch bevor eine Intubation vorbereitet und die entsprechenden Medikamente aufgezogen werden konnten, war die Nadelung spezifischer Akupunkturpunkte in wenigen Augenblicken erfolgt. Der YNSA-YinBasalganglien-Punkt [4] wurde dabei zusammen mit dem Punkt Renzhong [5] akupunktiert. Zusätzlich wurde der Punkt Qiangu akupunktiert [6].

Unmittelbar nach Nadelung des Qiangu war es direkt möglich, den Unterkiefer nach vorne zu bewegen und die oberen und unteren Atemwege mit einem Absaugkatheter freizumachen. Weitere supportive Maßnahmen hinsichtlich Atmung und Kreislauf waren während des gesamten Transports nicht mehr erforderlich. Der Patient atmete von diesem Zeitpunkt an spontan ohne weiteres Krampfen.

Durch eine intensive Stimulation des YNSA-Basalganglien-Punkts und einer ebenfalls intensiven Stimulation des Punktes Renzhong konnten die Krämpfe ohne eine weitere Gabe von Rivotril oder anderer Medikamente behandelt werden.

Der Transport verlief insgesamt komplikationslos. 
Um 23:45 Uhr wurde der Patient weiterhin bewusstlos mit einem systolischen Blutdruck von $100 \mathrm{~mm} \mathrm{Hg}$ und einer Herzfrequenz von 84/min, Sauerstoffsättigung 99\%, an die neurologische Abteilung eines universitären Lehrkrankenhauses übergeben.

\section{Diskussion}

Akupunktur und auch Akupressur werden in der Akutbehandlung bei Indikationen wie Asthma, akutem Harnverhalt, akutem apoplektischem Insult, Schock, Bewusstlosigkeit [7], allergischen Reaktionen auf Insektenstiche, Lippenödem, Kieferklemme, psychiatrischen Erkrankungen wie Manie, Unruhezuständen, Ängsten sowie akuten Schmerzen und Epilepsie angewendet [8-10]. Auch in der Militärmedizin und in Krisenregionen kommt Akupunktur zum Einsatz [11, 12].

Richard Umlauf hatte 1994 in seinem Buch «Akupunktur in der Notfallmedizin» [13] Erfahrungen aus 140000 Notfallbehandlungen mit Akupunktur an der Universitätsklinik in Brünn, Tschechien, in den Abteilungen Anästhesie, Chirurgie, Innere Medizin und Neurologie zusammengefasst. Für insgesamt 41 verschiedene Notfallindikationen empfiehlt er den supportiven oder alleinigen Einsatz von Akupunktur.

Stark eingeschränkte Mundöffnung oder Kieferklemme (Ankylostoma) kann sowohl myogene als auch arthrogene und neurogene Ursachen haben. Im Fall der Kieferklemme ist die Intubation erheblich erschwert. Im stationären Bereich stehen dann verschiedene Hilfen wie z.B. die fiberoptische Intubation oder die Intubation mit einem Bronchoskop zur Verfügung, die präklinisch nicht genutzt werden können. Hier bietet sich der versuchsweise Einsatz der Akupunktur mit dem Qiangu und dem Kiefergelenkspunkt der Ohrakupunktur als Hilfe für die präklinische Notfallsituation an.

Akupunktur könnte in der Zukunft supportiv auch gerade dort zum Einsatz kommen, wo konventionelle Therapieoptionen an ihre Grenzen stoßen.

Nach den neuesten Leitlinien der Arbeitsgemeinschaft der Wissenschaftlichen Medizinischen Fachgesellschaften (AWMF) [14] wird folgendes Vorgehen bei der prästationären Behandlung der Epilepsie empfohlen: Mittel der ersten Wahl ist nach Anlage eines intravenösen Zugangs nach wie vor das Benzodiazepin Clonazepam [15].

$\mathrm{Zu}$ den Erstmaßnahmen außerhalb der Klinik gehört auch die rektale Gabe von Diazepam (10 oder $20 \mathrm{mg}$ ) durch den Laien oder Rettungssanitäter. Hierdurch kann wertvolle Zeit bis zum Eintreffen des Notarztes gewonnen werden.

Im vorliegenden Fall - so vermuten die Autoren - hat anscheinend der Einsatz der Akupunktur allein eine rasche Lösung der drängenden Probleme erbracht. Durch die Verlegung des Nasenraums mit zähem Sekret war eine Beatmung über die Nase unmöglich. Eine Präoxygenierung vor der
Gabe von Medikamenten und vor einer Intubation war ebenfalls nicht möglich. Im schlimmsten Fall wäre eine Koniotomie notwendig geworden.

Da sowohl das Sistieren der Myoklonien als auch das Öffnen des Mundes ganz unmittelbar auf die Akupunkturintervention folgten, sehen wir in diesem Einzelfall einen direkten Zusammenhang zwischen Akupunktur und Behandlungseffekt. Insgesamt erhielt der Patient $2 \times 500 \mathrm{ml}$ Ringer-Lösung, aber keine zusätzlichen Medikamente. Bis zum letztendlichen Eintreffen in der Klinik hatte der Patient keine weiteren Krampfanfälle mehr, blieb aber tief bewusstlos. Bei Einsatzende betrug die GCS weiterhin 3 Punkte.

Es kann jedoch nicht bewiesen werden, dass die Akupunktur allein die beschriebenen Effekte hervorgerufen hat. Denkbar wäre auch eine verzögerte Nachwirkung des Rivotrils oder ein spontanes Sistieren des Anfalls. Hätte der Patient nicht so rasch auf die Akupunkturbehandlung angesprochen, wäre selbstverständlich sofort eine leitliniengerechte Therapie in Form einer erneuten Gabe von Rivotril sowie Intubation und Beatmung bei persistierendem Atemstillstand erfolgt.

Wir denken, dass selbst die technisch hochentwickelte Notfallmedizin durch Komplementärmedizin bereichert werden kann. Dies geht auch aus verschiedensten Falldarstellungen aktiv tätiger Notärzte [16-19] hervor. Die Akupunktur versteht sich als Zusatz zur etablierten Medizin, d.h. die Behandler wählen sorgsam nach strenger Indikationsstellung die Akupunktur als ergänzendes oder alternatives Heilverfahren, wobei dem Einsatz der konventionellen Medizin immer die oberste Priorität eingeräumt wird.

Anhänger aller medizinischen Fachrichtungen haben ein gemeinsames Ziel: leidenden Menschen zu helfen und klinische Probleme zu lösen [20]. Das mögliche Potenzial der Akupunktur in der Notfallmedizin, klinische Probleme zu lösen, kann derzeit nicht abschließend beurteilt werden, da umfangreiche kontrollierte Studien zum Einsatz von Akupunktur in der Notfallmedizin fehlen.

\section{Schlussfolgerung}

Aufgrund vieler positiver Erfahrungen mit Akupunktur in der Notfallmedizin - wie in diesem Fall bei der Behandlung eines epileptischen Anfalls mit Atemstillstand - möchten wir anregen, diese unter anderem wegen des raschen Wirkeintritts verstärkt supportiv sowohl im ärztlichen Notdienst wie auch im Notarztdienst einzusetzen.

\section{Disclosure Statement}

Die Autoren erklären, dass keine Interessenkonflikte bestehen. 


\section{Literatur}

1 Janssen M: Ausbildung und rechtliche Stellung von Rettungsdienstpersonal: Deutschland im internationalen Vergleich. Rettungsdienst 2007;6:610-615

2 Dick WF: Anglo-American vs. Franco-German Emergency Medical Services System. Prehosp Disaster Med 2003;18:29-37.

3 Schockert T: Was ist möglich mit YNSA?: - Expertenbefragung. DZA 2009;3:34-49.

4 Yamamoto T, Yamamoto H, Yamamoto MM: YNSA - Yamamoto Neue Schädelakupunktur Bad Kötzting, Verlag für Ganzheitliche Medizin, 2005, pp 66-67.

5 Deadman P, Al-Khafaji M, Baker K: Großes Handbuch der Akupunktur. Bad Kötzting, Verlag für Ganzheitliche Medizin, 2002, pp 593-594.

6 Gleditsch JM, Ogal HP: MAPS: Mikro-Aku-PunktSysteme. Stuttgart, Hippokrates, 2002, pp 187.

7 Streiberger K, Gries A: Acupuncture in diagnosing prehospital unconsciousness. Am J Emerg Med 2005;23:90-91.
8 Schockert T: YNSA im Rettungsdienst. DZA 2008;4:21-29.

9 Barker R, Kober A, Hoerauf K, Latzke D, Adel S, Kain ZN, Wang SM: Out-of-hospital auricular acupressure in elder patients with hip fracture: a randomized double blind trial. Acad Emerg Med 2006; 13:19-23.

10 Schockert T: Integration von Yamamoto Neuer Schädelakupunktur (YNSA) in die Notfallmedizin - Falldarstellung zur Analgesie mittels YNSA. DZA 2010;4:34-37.

11 Christine MH, Goerzt DC, Niemtzow CR, Burns SM, Fritts MJ, Crawford CC, Jonas WB: Auricular acupuncture in the treatment of acute pain syndromes: a pilot study. Mil Med 2006;171:1010-1014.

12 Niemtzow CR, Gambel J, Helms J, Pock A, Burns SM, Baxter J: Integrating ear and scalp acupuncture techniques into the care of blast-injured United States military service members with limb loss. J Altern Complement Med 2006;12:596-599.
13 Umlauf R: Akupunktur in der Notfallmedizin. Heidelberg, Haug 1994, pp 109-110.

14 www.awmf.org/leitlinien/aktuelle-leitlinien.

15 Brokmann J, Rossaint R: Repetitorium Notfallmedizin. Heidelberg, Springer, 2008, pp 364

16 Fleckenstein J, Schottdorf J, Kreimeier U, Irnich D: Akupunktur in der Notfallmedizin. Ergebnisse einer Fallstudie. Anästhesist 2011;9:1-7.

17 Neuhauser S, Schockert T: Erfolgreicher Einsatz von Akupunktur in der Notfallmedizin. ZTCM 2010;2:86-90.

18 Schockert T, Schulé S: Anwendung von Akupunktur in Notfallsituationen: Ergebnisse einer Umfrage im deutschsprachigen Raum. DZA 2011;3: 12-16.

19 Schulé S: Akupressur bei Morbus Menière - effektive Hilfe dank sanftem Druck. Rettungs-Magazin 2012;1:31

20 Walach $\mathrm{H}$ : The campaign against CAM - a reason to be proud. J Hol Healthcare 2009;6:8-13. 